\title{
Nonequilibrium thermodynamics of an interface
}

\author{
Marco Schweizer, ${ }^{1}$ Hans Christian Öttinger, ${ }^{1, *}$ and Thierry Savin ${ }^{1,2, \dagger}$ \\ ${ }^{1}$ Department of Materials, ETH Zürich, CH-8093 Zürich, Switzerland \\ ${ }^{2}$ Department of Engineering, University of Cambridge, Cambridge CB2 1PZ, United Kingdom
}

(Dated: April 8, 2016)

\begin{abstract}
Interfacial thermodynamics has deep ramifications in understanding the boundary conditions of transport theories. We here present a formulation of local equilibrium for interfaces that extends the thermodynamics of the "dividing surface," as introduced by Gibbs, to nonequilibrium settings such as evaporation or condensation. By identifying the precise position of the dividing surface in the interfacial region with a gauge degree of freedom, we exploit gauge-invariance requirements to consistently define the intensive variables for the interface. The model is verified under stringent conditions by employing high-precision nonequilibrium molecular dynamics simulations of a coexisting vapor-liquid Lennard-Jones fluid. We conclude that the interfacial temperature is determined using the surface tension as a "thermometer," and can be significantly different from the temperatures of the adjacent phases. Our findings lay new foundations for nonequilibrium interfacial thermodynamics.

PACS numbers: 05.70.Ln, 68.35.Md, 02.70.Ns
\end{abstract}

\section{INTRODUCTION}

Interfacial effects can be of paramount importance for transport and thermal processes in heterogeneous systems, and their account is central to several areas of physics (surface and colloid science, capillarity, optics), chemistry (heterogeneous reaction, adsorption), biology (membrane trafficking, transduction) and engineering (materials science, interfacial rheology) [1-6]. Consequently, many efforts have been made in the last century to build a rigorous, thermodynamically admissible description of interfaces at equilibrium and at nonequilibrium $[1-3,5]$. Broadly, we may separate the strategies used so far into two complementary categories: the smooth - or diffuse - interface approach, and the sharp interface approach [3-5].

The diffuse interface model examines continuous profiles of local densities and compositions, that exhibit large but bounded gradients from one phase to the other across an interfacial layer of finite thickness. The corresponding groundwork for models was laid by van der Waals [6-8] in terms of gradient theories, and further implemented by Korteweg [9] and Cahn and Hilliard [10]. The associated equations can be integrated numerically, and many interfacial systems have been investigated using the resulting popular "phase field" models [11-13]. It is however challenging to experimentally access the resolution required for measuring smooth profiles of thermodynamic quantities in the vicinity of the interface. Hence, the validation of the aforementioned continuous approaches by experiments is often done indirectly, by relying on the observations of mesoscopic patterns of structures and dynamics that are strongly influenced by interfacial effects [14-17]. Nevertheless, the diffuse interface model is, by nature, not well adapted for assessing macroscopic phenomena, at which scale the boundaries of bulk phases are better modeled by sharp interfaces $[3,5]$.

\footnotetext{
*hco@mat.ethz.ch

$\dagger$ t.savin@eng.cam.ac.uk
}

The precursors to this strictly two-dimensional model can be attributed to Gibbs [18], who first carried out the thermodynamic description of what he named the "dividing surface". His description relies on so-called excess densities, which are thermodynamic extensive quantities assigned to the interface, considered itself as a 2D autonomous thermodynamic system $[3,6]$, and whose temperature and chemical potential are the ones shared with the bulk phases in equilibrium. Other intensive parameters for the interface, such as its surface tension or bending rigidity, can be derived from microstructural models [19-24]. Their study is the attention of interfacial rheology [5], where Gibbs' description is indeed of central importance: most interfacial constitutive equations are established in the sharp interface framework, and two-dimensional equivalents of bulk models, such as the Boussinesq, Maxwell or Voigt relations, are routinely used to describe interfacial rheometry $[1,3,5]$. Apart from rheology, the compliance of Gibbs adsorption isotherms [25], or the description of the Marangoni effect [26] may also be seen as celebrations of Gibbs' model of interfacial thermodynamics. But it is the ubiquitous use of surface boundary conditions in transport theories that gives an even more compelling reason for the fundamental role and appeal of a sharp interface approach. Yet all these applications have mostly nonequilibrium settings, where thermodynamic fields may not be uniform across the system, and for which Gibbs' description is, therefore, not suited.

To study a nonequilibrium dividing surface, one may exploit the relation between the sharp and diffuse treatments of an interface. The excess quantities used by Gibbs can indeed be defined from the density profiles considered with a smooth interface. However, due to additional gradient terms and intrinsic anisotropies in the interfacial region, the local free energy is irremediably biased from its bulk thermodynamic definition. This matter greatly challenges the central paradigm of nonequilibrium thermodynamics: the local equilibrium assumption, and the corresponding local definition of intensive quantities $[6,27]$. Several attempts have been made to address the local equilibrium of interfaces [27-36]. However, reconciling the diffuse and sharp interface approaches into a thermodynamically admissible description is ultimately con- 
fronted to two fundamental problems: first, how to locate the Gibbs dividing surface in the interfacial region of the smooth density profiles; second, how to define an interfacial temperature and chemical potential in nonequilibrium [37]. The first issue is a well-known and striking paradox: thermodynamics of interfaces cannot depend on the observer's choice of a surface even though the extensive excess densities strongly depend on this choice. Solving the second issue, although not encountered at equilibrium, is of utmost importance: it is a central requirement for extending local equilibrium models to interfaces, and to thus develop 2D nonequilibrium thermodynamics.

We recently suggested a consistent description of twodimensional interfaces that addresses these issues using (i) a formalism based on gauge transformations associated with changing the location of the dividing surface, and (ii) the surface tension as the interfacial thermometer [37]. After briefly explaining our model for a single-component biphasic fluid, we employ extensive, high-precision nonequilibrium molecular dynamics simulations of a vapor-liquid system to validate our description.

\section{THEORY}

Let us consider a single-component system with two phases separated by a planar dividing surface. Calling $x$ the coordinate normal to the interface, we write $u(x), s(x)$ and $\rho(x)$ the continuous densities of internal energy, entropy and mass, respectively. To calculate the excess mass density $\rho^{\mathrm{s}}$, we separate the variations $\rho^{\alpha}(x)$ and $\rho^{\beta}(x)$ of $\rho(x)$ in each phase $\alpha$ and $\beta$ (let $\alpha$ be for smaller $x$ ) outside the interfacial layer. Upon choosing the precise location $x^{\mathrm{s}}$ of the plane dividing the phases within the interfacial region, we then define the "sharpened" density profile $\rho^{\alpha, \beta}\left(x, x^{\mathrm{S}}\right)=\rho^{\alpha}(x) \Theta\left(x^{\mathrm{S}}-x\right)+$ $\rho^{\beta}(x) \Theta\left(x-x^{\mathrm{s}}\right)$, with the step function $\Theta(x)=1$ for $x \geq 0$, 0 otherwise. The sharpening procedure thus requires the extrapolation of $\rho^{\alpha}(x)$ and $\rho^{\beta}(x)$ over the interfacial region (in equilibrium, $\rho^{\alpha}$ and $\rho^{\beta}$ are constant and their extrapolation is trivial). The excess mass density is then

$$
\rho^{\mathrm{s}}\left(x^{\mathrm{s}}\right)=\int\left[\rho(x)-\rho^{\alpha, \beta}\left(x, x^{\mathrm{s}}\right)\right] \mathrm{d} x,
$$

and similarly for $u^{\mathrm{s}}$ and $s^{\mathrm{s}}$, the excess densities of internal energy and entropy, respectively (see Fig. 2). Hence, all the excess densities depend on $x^{\mathrm{s}}$, chosen a priori arbitrarily. This degree of freedom is the one "apparently" lost with phase coexistence, upon invoking the Gibbs phase rule. The first issue is to ensure that interfacial thermodynamics is independent of the observer's choice for a dividing surface, even though the excess densities depend on it.

When in contact with nonequilibrium bulk phases, interfaces quickly relax to states that can locally be described by the same set of variables as equilibrium interfaces, with the same thermodynamic relations between these variables. This rationale for the interfacial local equilibrium leads us to hypothesize a generalization of Euler and Gibbs-Duhem rela- tions for the nonequilibrium interface,

$$
\begin{aligned}
u^{\mathrm{s}} & =T^{\mathrm{s}} s^{\mathrm{s}}+\gamma+\mu^{\mathrm{s}} \rho^{\mathrm{s}}, \\
0 & =s^{\mathrm{s}} d T^{\mathrm{s}}+d \gamma+\rho^{\mathrm{s}} d \mu^{\mathrm{s}},
\end{aligned}
$$

where the temperature $T^{\mathrm{s}}$ and the chemical potential $\mu^{\mathrm{s}}$ must now be defined for the autonomous interface with surface tension $\gamma$. The second issue is then to ascribe the intensive quantities $T^{\mathrm{s}}$ and $\mu^{\mathrm{s}}$ to such a $2 \mathrm{D}$ object.

We recently used the formalism of gauge transformation to address the two problems emphasized above [37]. The gauge is the choice of $x^{\mathrm{s}}$, and the gauge transformation $x^{\mathrm{s}} \rightarrow x^{\mathrm{s}}+\ell$ offsets $x^{\mathrm{s}}$ by a small displacement $\ell$ towards the phase $\beta$ and changes the densities to the new values, $u^{\mathrm{s}} \rightarrow u^{\mathrm{s}}+\ell \Delta u$, $s^{\mathrm{s}} \rightarrow s^{\mathrm{s}}+\ell \Delta s$ and $\rho^{\mathrm{s}} \rightarrow \rho^{\mathrm{s}}+\ell \Delta \rho$. Here, we have called $\Delta u=u^{\beta}-u^{\alpha}, \Delta s=s^{\beta}-s^{\alpha}$ and $\Delta \rho=\rho^{\beta}-\rho^{\alpha}$ the jumps of the corresponding bulk densities across the interface, assumed to be independent of the gauge $x^{\mathrm{s}}$. The intensive properties of the interface, $T^{\mathrm{s}}, \mu^{\mathrm{s}}$ and $\gamma$, must be gauge-invariant observables, and only one of them sets the state of the interface. Since $\gamma$ can be extracted unambiguously at both equilibrium and nonequilibrium (see later), we suggested to use the surface tension as the thermometer of the interface: $\gamma(T)$ is first tabulated at equilibrium, where $T$ is the uniform bulk temperature, and then $T^{\mathrm{s}}$ is deduced from the value of $\gamma$ in nonequilibrium. We then recommended that the interfacial chemical potential follows $\mu^{\mathrm{s}}=\mu\left(T^{\mathrm{s}}\right)$ from the equilibrium chemical potential. To address the ambiguity in the choice of $x^{\mathrm{s}}$, we expressed the condition that the Euler and Gibbs-Duhem equations must be gauge-invariant by $\Delta u=T^{\mathrm{s}} \Delta s+\mu^{\mathrm{s}} \Delta \rho$ and $0=\Delta s d T^{\mathrm{s}}+\Delta \rho d \mu^{\mathrm{s}}$, respectively. These equations are equivalent to the Clapeyron's relationships

$$
\begin{aligned}
\frac{d\left(\mu^{\mathrm{s}} / T^{\mathrm{s}}\right)}{d\left(1 / T^{\mathrm{s}}\right)} & =\frac{\Delta u}{\Delta \rho}, \\
-\frac{d \mu^{\mathrm{s}}}{d T^{\mathrm{s}}} & =\frac{\Delta s}{\Delta \rho},
\end{aligned}
$$

which, if indeed proven to be generalizable to nonequilibrium, would allow us to write Eqs. (2) into the equivalent forms:

$$
\begin{aligned}
& u^{\mathrm{s}}\left(T^{\mathrm{s}}, \rho^{\mathrm{s}}\right)-\rho^{\mathrm{s}} \frac{\Delta u}{\Delta \rho}=\frac{d\left(\gamma / T^{\mathrm{s}}\right)}{d\left(1 / T^{\mathrm{s}}\right)}, \\
& s^{\mathrm{s}}\left(T^{\mathrm{s}}, \rho^{\mathrm{s}}\right)-\rho^{\mathrm{s}} \frac{\Delta s}{\Delta \rho}=-\frac{d \gamma}{d T^{\mathrm{s}}} .
\end{aligned}
$$

We call these two equations (4) the "structural relationships" of the interface, for reasons that we now discuss.

In the gauge for which $\rho^{\mathrm{s}}=0$, defining the "equimolar dividing surface" (see Sec.IV A), Eqs. (4) simplify to highlight the role of the surface tension in our description: it is the thermodynamic potential of the interface, whose entropy and energy are obtained by separating the entropic and energetic contribution of $\gamma$ using its temperature dependence (see further discussions on that point in Sec. IV D). Hence, $\gamma$ characterizes the inherent structure of the interface. The additional terms arising in $s^{\mathrm{s}}$ and $u^{\mathrm{s}}$ upon a different choice of gauge are given in terms of the chemical potential $\mu^{\mathrm{s}}$, which rather characterizes coexistence. Our reasoning to define $T^{\mathrm{s}}$ from $\gamma$ relies 
on the structural autonomy of the interface: the interfacial region is not merely a transition zone for particles escaping one phase to join the other. Such non-autonomous interpretation of the interface would correlate its thickness with the traveling distance required by a particle for encountering enough collisions so as to join a well established phase. In the vaporliquid system investigated in the next sections, this correlation does not exist: the width $\omega$ of the interfacial region increases with temperature, whereas the width of the Knudsen layer of evaporation scales as the mean free path in the vapor, which decreases with the temperature.

We finish this section by noting that Öttinger and Venerus [38] recently suggested the following gauge invariance condition for the Euler equation: $\Delta u+\frac{1}{2}(\Delta \mathbf{v})^{2} /\left(\Delta \frac{1}{\rho}\right)=T^{\mathrm{s}} \Delta s+\mu^{\mathrm{s}} \Delta \rho$, where $\Delta \mathbf{v}=\mathbf{v}^{\beta}-\mathbf{v}^{\alpha}$ is the difference between the velocity fields of each phase, and $\Delta \frac{1}{\rho}=\frac{1}{\rho^{\beta}}-\frac{1}{\rho^{\alpha}}$. The additional term originates from possible flows in the bulk phases, which induce a transfer of kinetic energy between phases upon changing the gauge. Here, only Eq. (3a) is modified to $\frac{d\left(\mu^{\mathrm{s}} / T^{\mathrm{s}}\right)}{d\left(1 / T^{\mathrm{s}}\right)}=$ $\frac{\Delta u}{\Delta \rho}-\frac{1}{2} \mathbf{v}^{\alpha} \cdot \mathbf{v}^{\beta}$ by this correction, provided with the conservation of momentum at an immobile interface $\rho^{\alpha} \mathbf{v}^{\alpha}=\rho^{\beta} \mathbf{v}^{\beta}$ (this is the only scenario investigated here). In our study however, this correction is negligible. In fact, the negligibility of kinetic energy effects might also be required to obtain consistency of the gauge invariance condition of Ref. 38 with the momentum balance at the interface derived in that paper.

In general, only the fields of thermodynamic densities $a$ that are defined in a fixed reference (such as $a=\rho, s$, $\mathbf{M}=\rho \mathbf{v}$ the momentum density, or $e=u+\frac{1}{2} \rho \mathbf{v}^{2}$ the total energy density) have surface excess densities that strictly gaugetransform as $a^{\mathrm{s}} \rightarrow a^{\mathrm{s}}+\ell \Delta a$. Along the same lines, Legendre transformations of these densities do not normally gaugetransform because their variations lead to forbidden changes of intensive, gauge-invariant variables. For example, with the static approximation $u^{\mathrm{s}} \rightarrow u^{\mathrm{s}}+\ell \Delta u$, the excess Helmholtz free energy $f^{\mathrm{s}}=u^{\mathrm{s}}-T^{\mathrm{s}} s^{\mathrm{s}}$ gauge-transforms according to $f^{\mathrm{s}} \rightarrow f^{\mathrm{s}}+\ell\left(\Delta u-T^{\mathrm{s}} \Delta s\right)$, but not according to $f^{\mathrm{s}} \rightarrow f^{\mathrm{s}}+\ell \Delta f$.

\section{NONEQUILIBRIUM SYSTEMS}

We now employ nonequilibrium simulations to validate our thermodynamics description of the interface, Eqs. (2) and their gauge-invariance, by verifying Clapeyron's Eqs. (3) and the local equilibrium Eqs. (4) with our proposed definitions for $T^{\mathrm{s}}$ and $\mu^{\mathrm{s}}$. Details of the numerical methods are given in the appendix. Briefly, we simulated a one-component Lennard-Jones fluid with both liquid and vapor phases in coexistence, $(\alpha, \beta)=(\mathrm{g}, \mathrm{l})$, by using molecular dynamics (Fig. 1A). Nonequilibrium situations were created for two fundamentally different transport processes: we studied systems with a heat flux or with a mass flux through the planar interface. The heat flux is imposed by tuning the temperatures of two thermostated regions in the vapor and the liquid. A mass flux is created by removing particles from one phase and simultaneously inserting them in the other phase, at a given swapping rate. The temperature gradients investigated here
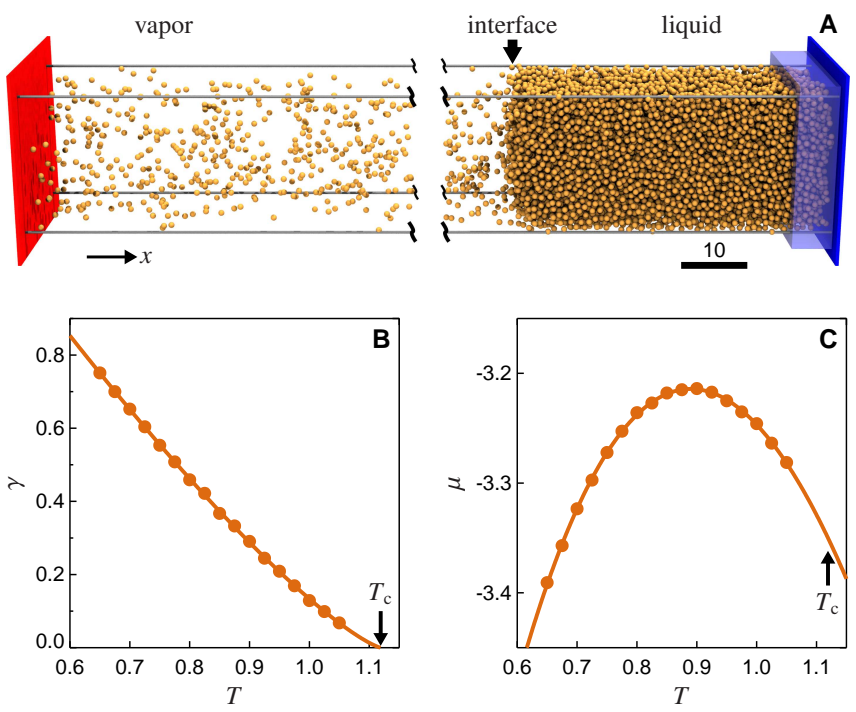

FIG. 1. (A) The simulations' unit cell is $213 \times 21 \times 21$ (in LennardJones unit; shown here truncated in the vapor region), periodically repeats in the $y, z$-directions and contains 16192 particles interacting through a spline-interpolated Lennard-Jones potential [39]. The reflecting boundaries in the $x$-direction (red in the vapor; blue in the liquid) and the blue volume are thermostated via velocity rescaling (see the appendix). (B) Equilibrium surface tension as a function of temperature; the data points follow the curve $\gamma(T)=\gamma_{0}\left(1-T / T_{\mathrm{c}}\right)^{v}$, where $T_{\mathrm{c}}$ is the critical temperature. (C) Equilibrium chemical potential as a function of temperature, fitted with the formula $\mu(T)=$ $\mu_{0}+a T \log T+b T$. Uncertainties in (B) and (C) are below $2 \%$ of the range of reported values.

are in the range $10^{6}-10^{8} \mathrm{~K} / \mathrm{m}$, and the speeds associated with the mass fluxes about $10 \mathrm{~m} / \mathrm{s}$ (see the appendix). In what follows, all quantities are given in Lennard-Jones units and calculated for the system's steady state.

The surface tension $\gamma$ of the interface was calculated by integrating the difference between the pressure components normal and tangential to the interface [20]. In all the cases investigated here, the anisotropy of the pressure tensor is localized at the interface, and a spatial integration of the components' difference does not depend on the position of the dividing surface. The surface tension is thus gauge-invariant. At equilibrium, an excellent fit of $\gamma(T)$ is $\gamma_{0}\left(1-T / T_{\mathrm{c}}\right)^{v}$, with $\gamma_{0}=2.2452, v=1.26$ and $T_{\mathrm{c}}=1.1192$ (Fig. 1B) [31]. The fit serves as the calibration rule to extract the interface temperature $T^{\mathrm{s}}=T_{\mathrm{c}}\left[1-\left(\gamma / \gamma_{0}\right)^{1 / v}\right]$ from the surface tension calculated in nonequilibrium situations.

Other specific atomistic expressions are time-averaged over steady state configurations to obtain $x$-dependent profiles of thermodynamic quantities. Fig. 2A and 2B show typical profiles of $\rho(x)$ and $u(x)$, respectively, in a nonequilibrium situation. Along with the stress tensor, $\rho$ and $u$ are mechanical quantities whose atomistic expressions are exact in the interfacial region. The chemical potential $\mu(x)$ is obtained by the Widom insertion method [40, 41]. In equilibrium, the uniform potential can be fitted by $\mu(T)=\mu_{0}+a T \log T+b T$ with $\mu_{0}=$ $-7.6510, a=-4.9849$ and $b=4.4042$ (Fig. 1C). In each bulk phase, the entropy density is calculated from the other profiles 
by invoking the Euler equation, $s(x)=\frac{u(x)}{T(x)}+\frac{p(x)}{T(x)}-\frac{\mu(x) \rho(x)}{T(x)}$, with $p(x)$ the isotropic pressure and $T(x)$ the kinetic temperature.

We note that our simulations are significantly more precise than previous works on nonequilibrium vapor-liquid systems [31, 39]. High-precision was required to discern small gauge changes and was achieved by two means. First, for each simulation, 80 times more data were acquired as compared to Ref. 31. Second, we eliminated any possible diffusion of the liquid film in the $x$-direction by "sticking" it to the thermostated wall instead of using the more common periodic boundaries (see the appendix).

\section{RESULTS AND DISCUSSIONS}

\section{A. Excess Densities}

To calculate the sharpened profile $\rho^{\mathrm{g}, \mathrm{l}}\left(x, x^{\mathrm{s}}\right)$, we fit the bulk variations of $\rho(x)$ with linear functions of $x$ (Figs. 2A and 2B). The resulting lines deviates from the actual profile over the interfacial width $\omega(\omega \sim 10$ in Fig. 2$)$, which is the minimum coarse-graining scale in the sharp interface approach. Higher order extrapolations may indeed be used [28] without significantly changing the results presented here, as long as these functions do not capture variation at length scales below $\omega$. We observe that $\rho^{\mathrm{s}}\left(x^{\mathrm{s}}\right)$ and $u^{\mathrm{s}}\left(x^{\mathrm{s}}\right)$ depend linearly on $x^{\mathrm{s}}$ (Fig. 2C), such that we may write

$$
\begin{aligned}
& \rho^{\mathrm{s}}\left(x^{\mathrm{s}}\right)=\Delta \rho\left(x^{\mathrm{s}}-x_{\rho}^{\mathrm{s}}\right), \\
& u^{\mathrm{s}}\left(x^{\mathrm{s}}\right)=\Delta u\left(x^{\mathrm{s}}-x_{u}^{\mathrm{s}}\right),
\end{aligned}
$$

with $\Delta \rho=\rho^{1}-\rho^{\mathrm{g}}$ and $\Delta u=u^{1}-u^{\mathrm{g}}$. Here $x_{\rho}^{\mathrm{s}}$ is the location of the "equimolar" interface such that $\rho^{\mathrm{s}}\left(x_{\rho}^{\mathrm{s}}\right)=0$, and $x_{u}^{\mathrm{s}}$ is the "equienergetic" interface for which $u^{\mathrm{s}}\left(x_{u}^{\mathrm{s}}\right)=0$. Importantly, there exist a non-negligible difference between $x_{\rho}^{\mathrm{s}}$ and $x_{u}^{\mathrm{s}}$ (see inset of Fig. 2C). The linearity of $\rho^{\mathrm{s}}$ and $u^{\mathrm{s}}$ with $x^{\mathrm{s}}$ indicates
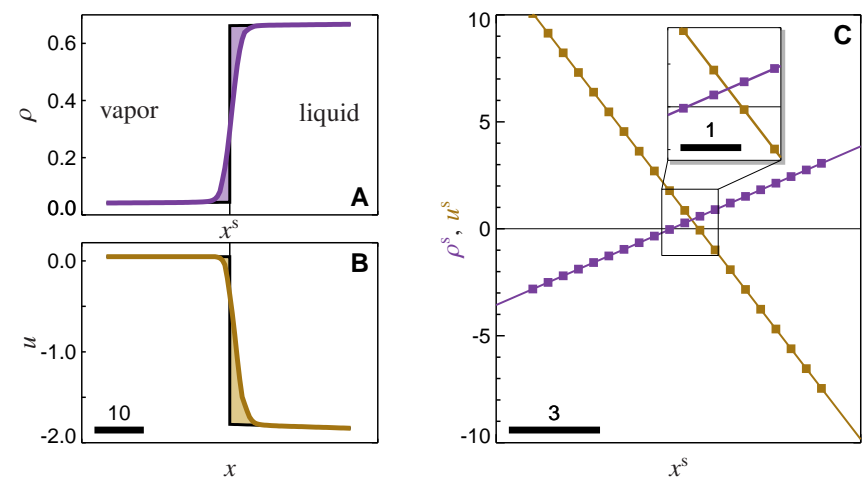

FIG. 2. (A-B) Profiles of mass (A) and internal energy (B) densities in the vicinity of the interface subjected to a heat flux; uncertainties are below $0.5 \%$ of the observed ranges. (C) The corresponding excess densities $\rho^{\mathrm{s}}$ and $u^{\mathrm{s}}$, calculated from (A) and (B), respectively, as the shaded area between the profiles (colored curves) and their sharpened extrapolations (black lines) for various positions $x^{\mathrm{s}}$ of the dividing surface (see Eq. (1)); uncertainties are below $1 \%$ of the range of values in this panel.
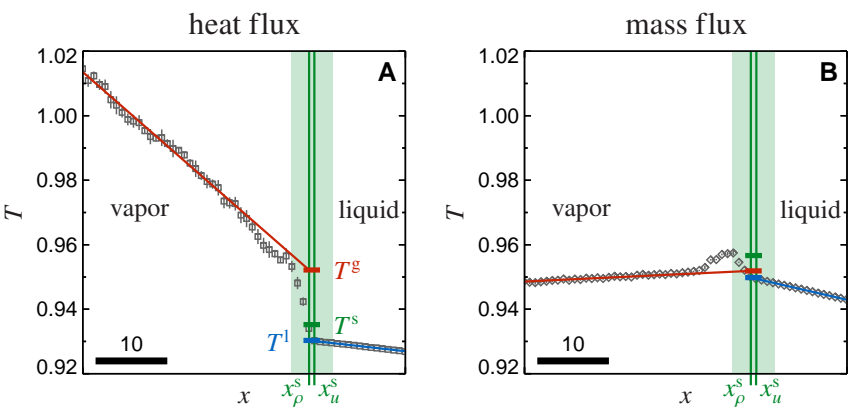

FIG. 3. Kinetic temperature profiles in the vicinity of the interface subjected to a heat flux (A) or a mass flux (B). The red and blue lines are linear extrapolation of the bulk profiles and used to define the liquid and vapor interfacial temperatures, as shown by the dashes and compared with $T^{\mathrm{s}}$ (green dash). The shaded area indicates the interfacial region, with the equimolar and equienergetic dividing surfaces shown by the green lines.

that $\Delta \rho$ and $\Delta u$ do not significantly depend on the interface location, meaning that they are gauge-invariant. We initially inferred this approximation in our theoretical arguments. It comes from the fact that the bulk densities do not vary significantly over distances comparable to $\omega$, even in the extreme conditions of nonequilibrium investigated here. Hence, $\rho^{\mathrm{g}}\left(x^{\mathrm{s}}\right)$ varies by only a few percent when moving $x^{\mathrm{s}}$ by 10 , as opposed to $\rho^{\mathrm{s}}\left(x^{\mathrm{s}}\right)$, which can even change sign when moving the interface by $\sim 1$.

We also observe slow variations of $s(x)$ in the bulk phases, allowing us to extract, without calculating $s^{\mathrm{s}}$, the interfacial jump $\Delta s=s^{1}-s^{\mathrm{g}}$ directly from $s^{\mathrm{l}}(x)$ and $s^{\mathrm{g}}(x)$ and independently of any extrapolated location chosen within the interfacial region. One may wish to write $s^{\mathrm{s}}$ using an expression similar to Eqs. (5), but an eventual "equientropic" gauge $x_{s}^{\mathrm{s}}$, for which $s^{\mathrm{s}}\left(x_{s}^{\mathrm{s}}\right)=0$, would inconveniently depend on the reference entropy. Augmenting this reference by $S_{0}$ translates $s^{\mathrm{s}}$ by $-S_{0} \rho^{\mathrm{s}}, \mu^{\mathrm{s}}$ by $S_{0} T^{\mathrm{s}}$ and $\Delta s$ by $-S_{0} \Delta \rho$. We thus prefer to express the explicit gauge dependence of $s^{\mathrm{s}}$ by also separating its reference-dependent component. Without loss of generality, we write

$$
s^{\mathrm{s}}\left(x^{\mathrm{s}}\right)=\Delta s\left(x^{\mathrm{s}}-x_{\rho}^{\mathrm{s}}\right)+\psi\left(T^{\mathrm{s}}\right),
$$

where $\psi\left(T^{\mathrm{s}}\right)=s^{\mathrm{s}}\left(x_{\rho}^{\mathrm{s}}\right)$ is a gauge-invariant thermodynamic function of the temperature only, and that does not depend on the reference entropy.

\section{B. Temperature Profiles}

Although the temperature calculated from the kinetic energy has the classical interpretation in both bulk phases, its meaning in the interfacial region is, admittedly, questionable. Nevertheless, Fig. 3 illustrates the conspicuous difficulty of defining a gauge-invariant interfacial temperature from such profile, since it exhibits sharp variations near the interface [42]. The profiles of temperatures, linearly extrapolated from the bulk phases, intercept neither at a definable location, nor 
at the interfacial temperature $T^{\mathrm{s}}$ advocated here. Therefore, a systematic macroscopic description of the system must allow discontinuous temperatures across its interface.

The equimolar and equienergetic dividing surfaces are shown in Fig. 3 to help outlining the interfacial region. For the mere purpose of illustrating our following arguments, $T^{\mathrm{g}}$ and $T^{1}$, the vapor and liquid temperatures at the interface, are extracted at $x^{\mathrm{s}}=x_{\rho}^{\mathrm{s}}$ (Fig. 3); but this choice has no effect on any of the conclusions in the present study.

\section{Clapeyron's Relationships}

Equipped with equilibrium and nonequilibrium values of $\Delta \rho, \Delta s$ and $\Delta u$, we can now check the validity of the nonequilibrium Clapeyron's relationships, Eqs. (3). To do so, we define the two coexistence temperatures, $T_{u}^{\text {coex }}$ and $T_{s}^{\text {coex }}$ that are solutions of Eqs. (3a) and (3b), respectively, for the observed jump ratios. These temperatures are uniquely defined using the fit $\mu(T)$. If $T=T_{u}^{\text {coex }}=T_{s}^{\text {coex }}$, then $T$ is the best temperature choice for the interface to verify the Clapeyron's relationships. Figures $4 \mathrm{~A}$ and $4 \mathrm{~B}$ report the relative deviations of $T^{\mathrm{s}}$ from $T_{u}^{\text {coex }}$ (Fig. 4A) and $T_{s}^{\text {coex }}$ (Fig. 4B) at equilibrium (where $T^{\mathrm{s}}$ is the system's uniform temperature) and at nonequilibrium (where $T^{\mathrm{s}}$ is set by $\gamma$ ). It also presents the deviations of the vapor and liquid temperatures $T^{\mathrm{g}}$ and $T^{\mathrm{l}}$. Our definition $T^{\mathrm{s}}$ consistently returns values that are, within the uncertainties, equal to the coexistence temperatures. In contrast, the vapor and liquid temperatures at the interface deviate from $T_{u}^{\text {coex }}$ and $T_{s}^{\text {coex }}$, and thus appear as inappropriate choices for the surface temperature.

We observe that $T^{\mathrm{s}}$ neither correlates with the magnitudes of the heat or the mass fluxes, which quantify how far from equilibrium the system is being pushed, nor with the temperature jump $\Delta T=T^{1}-T^{\mathrm{g}}$. Hence, discontinuities in temperature may also exist for moderate fluxes across the interface [43-45]. We verified however that despite the apparent scatter of the bulk temperatures in Fig. $4 \mathrm{~A}, \Delta \frac{1}{T}$ is proportional to the fluxes through the Kapitza resistances, which are indeed monotonic functions of $T^{\mathrm{s}}$ (data not shown) [46]. Nevertheless, our description does not require to regard the fluxes as independent variables of the interfacial thermodynamics [47].

\section{Structural Relationships}

We now assess the validity of the structural relationship Eq. (4a), which can be expressed in terms of the gauge difference

$$
\Delta u\left(x_{\rho}^{\mathrm{s}}-x_{u}^{\mathrm{s}}\right)=\gamma\left(T^{\mathrm{s}}\right)-T^{\mathrm{s}} \gamma^{\prime}\left(T^{\mathrm{s}}\right),
$$

by using Eqs. (5) and $\gamma^{\prime}=\frac{d \gamma}{d T}$. We proceed as before by defining a structural temperature $T_{u}^{\text {stru }}$ as the unique solution of Eq. (6), and verify that the relative deviations of our choice of $T^{\mathrm{s}}$ from $T_{u}^{\mathrm{stru}}$ vanishes (within uncertainties) for all situations investigated here (Fig. 4C). This agreement is remarkable, both in equilibrium and nonequilibrium, despite the fact
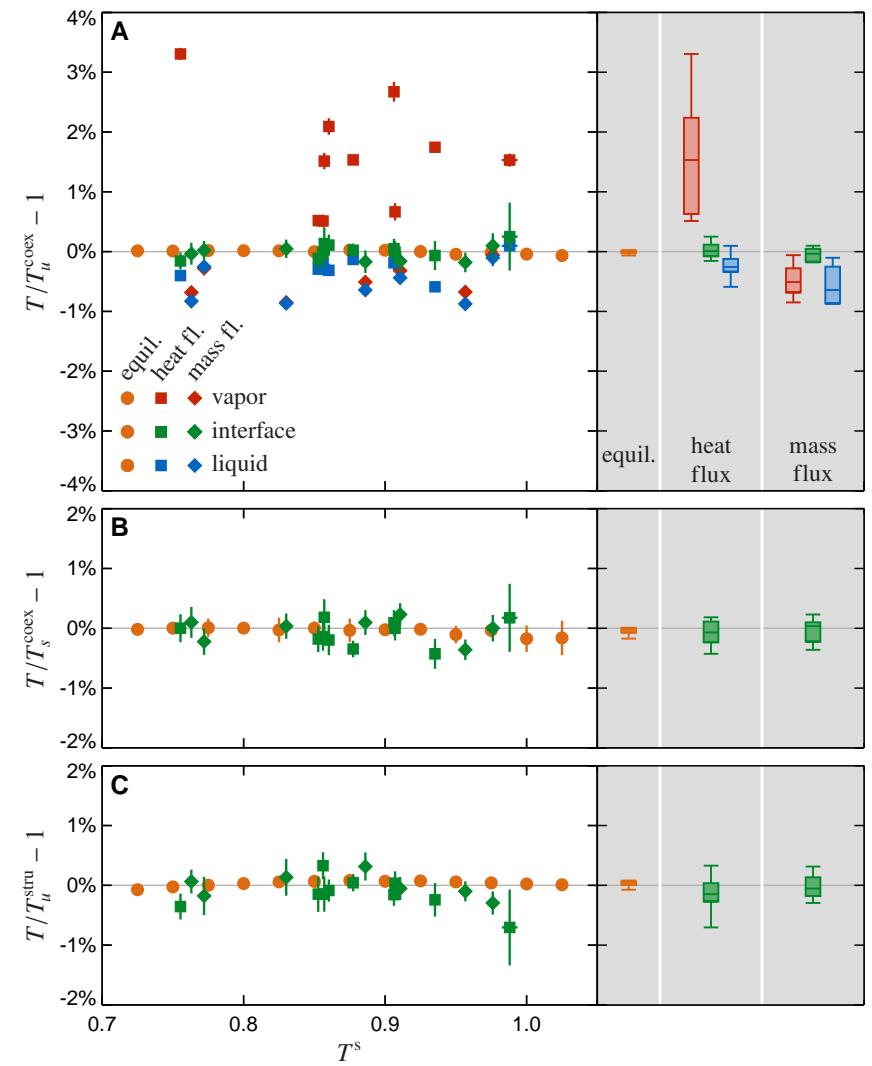

FIG. 4. (A) Relative deviations of the vapor, liquid and interface temperatures from the values $T_{u}^{\text {coex }}$ for which Eq. (3a) is verified. (B) and (C) are, respectively, the relative deviations of $T^{\mathrm{s}}$ from the temperature $T_{s}^{\text {coex }}$ that verifies Eq. (3b), and from the temperature $T_{u}^{\text {stru }}$ that verifies Eq. (4a). On the right side of each panel, the grey chart shows the corresponding box-and-whisker diagram in each thermodynamics situation investigated here.

that Eq. (6) initially results from macroscale thermodynamic arguments and that $x_{u}^{\mathrm{s}}-x_{\rho}^{\mathrm{s}}$ is of the order of the molecular distances, that is, much smaller than $\omega$.

In a similar manner, we can use Eqs. (5a) and (5c) to express the structural relationship Eq. (4b) as

$$
\psi\left(T^{\mathrm{s}}\right)=-\gamma^{\prime}\left(T^{\mathrm{s}}\right) .
$$

The direct validation of this equality requires a way to calculate $s^{\mathrm{s}}$ that does not rely on thermodynamic relationships (which would inevitably assume a priori the local equilibrium of the interface). Although we could not implement such a method, we note that we have already proved $\gamma\left(T^{\mathrm{s}}\right)=$ $u^{\mathrm{s}}+T^{\mathrm{s}} \gamma^{\prime}\left(T^{\mathrm{s}}\right)$ (from Eq. (6)) and $s^{\mathrm{s}}=\psi\left(T^{\mathrm{s}}\right)$ (from Eq. (5c)) in the equimolar gauge. This choice of gauge precludes any mass variation for the interface, and $\gamma$ then accounts for the Helmholtz free energy excess density of the interface as the amount of work per unit area that can be collected from it. Consequently, for $x^{\mathrm{s}}=x_{\rho}^{\mathrm{s}}$, we can write $\gamma\left(T^{\mathrm{s}}\right)=u^{\mathrm{s}}-T^{\mathrm{s}} s^{\mathrm{s}}$ which identifies $\psi\left(T^{\mathrm{s}}\right)=-\gamma^{\prime}\left(T^{\mathrm{s}}\right)$, thus proving Eq. (7). Note that $\gamma^{\prime}\left(T^{\mathrm{s}}\right)$ is a gauge-invariant quantity that does not depend on the reference entropy, in accord with the requirements for $\psi\left(T^{\mathrm{s}}\right)$. We have now validated Eqs. (2) and their gauge- 
invariance for a nonequilibrium vapor-liquid system where the interface has its temperature and chemical potential solely set by its surface tension.

\section{CONCLUSIONS}

For a single-component fluid with vapor-liquid coexisting phases, the surface tension $\gamma(T)$ is the only observable required to establish the complete state of the planar interface: under constant $\gamma$, the interface will have the same intrinsic structure as in equilibrium, even if it is separating nonequilibrium phases. Stemming from this intuitive concept, and by relying on gauge transformation arguments, we were able to verify the local equilibrium approximation for a $2 \mathrm{D}$ thermodynamic system. The precise location of the corresponding dividing surface may be chosen by convenience, without altering the thermodynamics.

We expect that these findings will help the development of interfacial nonequilibrium thermodynamics, even if they here concern a rather idealized situation. To extend the applicability of our model, future studies should notably consider multicomponent systems, curved interfaces [38] and other coexisting phases. Nevertheless, our definition of the interface temperature, using the surface tension as the thermometer, already offers a new and practical asset to experimentalists. For a typical monoatomic fluid such as argon (see the appendix), the liquid and vapor temperatures may differ from the surface temperature by a few degrees in nonequilibrium settings. Not only such differences should indeed be detectable using conventional instrumentations, they also may have heavy consequences when establishing constitutive models of interfaces. Notably, in a mass-conversion process such as condensation (or evaporation), the surface temperature can even be higher (or lower) than the surrounding bulk temperatures.

\section{Appendix: Numerical Methods}

We simulated a fluid of particles interacting through the smoothed Lennard-Jones spline potential, expressed at a separating distance $r$ by

$$
\phi(r)= \begin{cases}4 \epsilon\left[\left(\frac{\sigma}{r}\right)^{12}-\left(\frac{\sigma}{r}\right)^{6}\right] & \text { if } 0<r<r_{\mathrm{s}} \\ \zeta_{1}\left(r-r_{c}\right)^{3}+\zeta_{2}\left(r-r_{c}\right)^{2} & \text { if } r_{\mathrm{s}} \leq r \leq r_{\mathrm{c}} \\ 0 & \text { otherwise }\end{cases}
$$

The cutoff radius is $r_{\mathrm{c}}=2.5 \times \sigma$ to account for the longrange Lennard-Jones potential, while the spline cutoff $r_{\mathrm{s}}=$ $\frac{48}{67} r_{\mathrm{c}}$ and the spline coefficients $\zeta_{1}=0.099194 \times \epsilon / \sigma^{3}$ and $\zeta_{2}=-0.16346 \times \epsilon / \sigma^{2}$ are chosen such that the potential and its first derivative are continuous. Typical values of $\sigma$ and $\epsilon$ for a monoatomic Lennard-Jones system, made of argon-like particles [35], are $\sigma=3.822 \times 10^{-10} \mathrm{~m}$ and $\epsilon=1.654 \times 10^{-21} \mathrm{~J}$, with a particle's mass $m=6.690 \times 10^{-26} \mathrm{~kg}$. In the main text, we set $\sigma=1, m=1, \tau=\left(\frac{m \sigma^{2}}{\epsilon}\right)^{1 / 2}=1$ and $\theta=\frac{\epsilon}{k_{\mathrm{B}}}=1$ to define the Lennard-Jones units of length, mass, time and temperature respectively.
We placed 16192 particles in a simulation box with dimensions $L_{x}=213 \sigma$ and $L_{y}=L_{z}=21 \sigma$ along the $x, y$ and $z$-axis, respectively. We used periodic boundary conditions in the $y$ and $z$ directions. At $x=0$ (in the vapor) and $x=L_{x}$ (in the liquid), we used thermostated reflecting boundary conditions: particles hitting those surfaces were reflected according to a Maxwell distribution to impose a specific temperature. The liquid thermostat was complemented by rescaling accordingly the velocities of the particles in the volume $L_{x}-6 \sigma<x<L_{x}$ (see Fig. 1A).

Newton's evolution equations used to obtain the trajectories $\left\{\mathbf{r}_{i}(t)\right\}$ of the particles were integrated using the velocity Verlet algorithm with a time step of $\delta t=0.0005 \tau$. A small force of magnitude $0.005 \frac{\epsilon}{\sigma}$ in the $x$ direction was further applied in the liquid thermostat to pin the liquid phase at the surface $x=L_{x}$. The system was preconditioned for $3 \times 10^{6} \delta t$, upon which a steady liquid film coexisting with its vapor formed (Fig. 1A).

To simulate a system with an imposed heat flux, we set the vapor thermostat to a higher temperature than the liquid one. To create a mass flux, a liquid particle $i$ located in $L_{x}-6 \sigma<x<L_{x}$ was instantaneously translated by $-\left(L_{x}-6 \sigma\right)$ in the $x$ direction with a tunable probability after each Verlet time step. The attempt to insert the particle was accepted with probability $\min \left\{1, \exp \left[-\frac{\Phi_{i}(t)}{k_{\mathrm{B}} T}\right]\right\}$, where, calling $r_{i j}(t)=\left|\mathbf{r}_{i j}(t)\right|=\left|\mathbf{r}_{i}(t)-\mathbf{r}_{j}(t)\right|, \Phi_{i}(t)=\sum_{j \neq i} \phi\left(r_{i j}(t)\right)$ is the total interaction energy of the particle at its new location (this step was necessary to avoid singularities in case the particle was placed too close to another one; for this reason, simulating evaporation - requiring particles reinsertion in the liquid - is prohibitively more time-consuming). We evolved the system for another $6 \times 10^{6} \delta t$ under the new constraints to generate the corresponding nonequilibrium stationary state. Table I gives the set of thermostats' temperatures and swapping probabilities of the 17 nonequilibrium simulations performed here.

We binned the simulation box into 426 layers of thickness $\sigma / 2$ in the $x$-direction. We calculated the instantaneous mass density in layer $k, \rho_{k}(t)=m n_{k}(t) / V_{\mathrm{b}}$, where $V_{\mathrm{b}}=\sigma L_{y} L_{z} / 2$ is the volume of a bin and the instantaneous number of particles in layer $k$ at time $t$ is $n_{k}(t)=\sum_{i} \mathbb{1}_{k i}(t)$, with $\mathbb{1}_{k i}(t)$ the following indicator function: $\mathbb{1}_{k i}(t)=1$ if the particle $i$ is in layer $k$ at time $t$ and $\mathbb{1}_{k i}(t)=0$ otherwise. The corresponding discrete mass density profile is then defined at the position $x_{k}$ of the layer $k$ by $\rho\left(x_{k}\right)=\bar{\rho}_{k}$, where $\bar{a}$ indicates the time average of the process $a(t)$ (calculated over $50 \times 10^{6} \delta t$ in equilibrium, $80 \times 10^{6} \delta t$ in nonequilibrium).

Similarly, the instantaneous internal energy density $u_{k}(t)$ in layer $k$ at time $t$ is given by $u_{k}(t)=\eta_{k}(t)+$ $\frac{1}{2 V_{\mathrm{b}}} \sum \sum_{i \neq j} \mathbb{1}_{k i}(t) \phi\left(r_{i j}(t)\right)$, where $\eta_{k}(t)=\frac{m}{2 V_{\mathrm{b}}} \sum_{i} \mathbb{1}_{k i}(t)\left[\mathbf{v}_{i}(t)-\right.$ $\left.\overline{\mathbf{v}}_{k}\right]^{2}$ is the instantaneous, non-convected kinetic energy. Here, $\mathbf{v}_{i}(t)=\dot{\mathbf{r}}_{i}(t)$ and $\mathbf{v}\left(x_{k}\right)=\overline{\mathbf{v}}_{k}$ indicates the mean speed of the fluid in layer $k$, obtained by time-averaging the instantaneous velocity $\mathbf{v}_{k}(t)=\frac{1}{n_{k}(t)} \sum_{i} \mathbb{1}_{k i}(t) \mathbf{v}_{i}(t)$ of the particles in layer $k$. The final profile of the internal energy density is given by $u\left(x_{k}\right)=\bar{u}_{k}$. There is an ambiguity in defining the instantaneous value of the internal energy of a layer, due to the potential energy contribution: if two interacting particles are in different layers, there are various ways of distributing their 
TABLE I. Nonequilibrium simulation conditions (thermostats' temperatures and swapping probabilities) in Lennard-Jones units.

\begin{tabular}{l|r|ccc}
\hline \hline Type & $\#$ & $\begin{array}{c}\text { Vapor } \\
\text { thermostat }\end{array}$ & $\begin{array}{c}\text { Liquid } \\
\text { thermostat }\end{array}$ & $\begin{array}{c}\text { Swapping } \\
\text { frequency }\end{array}$ \\
\hline & 1 & 1.025 & 0.830 & 0 \\
& 2 & 1.025 & 0.840 & 0 \\
& 3 & 1.050 & 0.750 & 0 \\
Heat & 4 & 1.125 & 0.890 & 0 \\
flux & 5 & 1.150 & 0.850 & 0 \\
& 6 & 1.200 & 0.870 & 0 \\
& 7 & 1.250 & 0.850 & 0 \\
& 8 & 1.300 & 0.920 & 0 \\
& 9 & 1.300 & 0.980 & 0 \\
& 10 & 1.350 & 0.900 & 0 \\
\hline & 11 & 0.740 & 0.740 & 0.00080 \\
Mass & 12 & 0.740 & 0.740 & 0.00135 \\
flux & 13 & 0.790 & 0.790 & 0.00135 \\
& 14 & 0.870 & 0.870 & 0.00080 \\
& 15 & 0.890 & 0.890 & 0.00080 \\
& 16 & 0.940 & 0.940 & 0.00080 \\
& 17 & 0.970 & 0.970 & 0.00025 \\
\hline \hline
\end{tabular}

potential energy between the two layers. Hence, the potential energy may be attributed to the layer in which the center of mass of the pair particles falls, it may be distributed proportionally between the layers, or each layer may be given one half of the potential energy. The latter choice corresponds to the formula given in the text. We found however that the variations associated with different choices in the definition of the local internal energy were orders of magnitude smaller than other sources of error.

The kinetic temperature profile is calculated as $T\left(x_{k}\right)=$ $\frac{2 m}{3 k_{\mathrm{B}}} \frac{\bar{\eta}_{k}}{\bar{\rho}_{k}}$, and the pressure as $p\left(x_{k}\right)=\bar{p}_{k}$ with $p_{k}(t)=\frac{2}{3} \eta_{k}(t)+$ $\frac{1}{6 V_{\mathrm{b}}} \sum \sum_{i \neq j} \mathbb{1}_{k i}(t) \mathbf{r}_{i j} \cdot \mathbf{F}_{i j}$, where $\mathbf{F}_{i j}=-\left.\frac{\mathbf{r}_{i j}}{r_{i j}} \frac{d \phi}{d r}\right|_{r=r_{i j}}$ is the force acting on particle $i$ due to particle $j$.

The surface tension $\gamma$ of the interface was calculated by summing the difference between the components of the pressure that are normal and parallel to the interface, $\gamma=$ $-\frac{1}{4 L_{x} L_{y}} \sum \sum_{i \neq j}\left(\mathbf{r}_{i j} \cdot \mathbf{F}_{i j}-3 \mathbf{n} \cdot \mathbf{r}_{i j} \mathbf{F}_{i j} \cdot \mathbf{n}\right)$, where $\mathbf{n}$ is the unit vector normal to the interface [20]. Figure 1B shows the variations of $\gamma$ as a function of $T$ at equilibrium.

The chemical potential $\mu$ in the system was obtained with the Widom insertion method [40, 41]. We first write the sum $\mu=\mu_{\mathrm{id}}+\mu_{\mathrm{ex}}$, where $\mu_{\mathrm{id}}=-\frac{3}{2} \frac{k_{\mathrm{B}} T}{m} \log \frac{T}{\theta}+\frac{k_{\mathrm{B}} T}{m} \log \frac{\rho \sigma^{3}}{m}$ is the chemical potential of an ideal gas with mass density $\rho$, at temperature $T$, and $\mu_{\mathrm{ex}}$ the additional term that takes intermolecular interactions into account and that is calculated with the insertion method. The latter consists in inserting a LennardJones particle in the system, say at a position $\mathbf{r}_{k}$ in the layer $k$ and calculating $\mu_{\mathrm{ex}, k}(t)=-\frac{k_{\mathrm{B}} T}{m} \ln \left\langle\exp \left[-\frac{\Phi_{k}(t)}{k_{\mathrm{B}} T}\right]\right\rangle$, where $\langle\ldots\rangle$ indicates an average over all possible trial insertions at this location [41]. We attempted 12 randomly generated trial insertions in each bin every time-step, from which we calculated the instantaneous values $\mu_{\mathrm{ex}, k}(t)$. We then performed a time-average to get $\mu_{\mathrm{ex}}\left(x_{k}\right)=\bar{\mu}_{\mathrm{ex}, k}$, and finally $\mu\left(x_{k}\right)=\mu_{\mathrm{id}}\left(x_{k}\right)+\mu_{\mathrm{ex}}\left(x_{k}\right)$, where $\mu_{\text {id }}$ inherits its $x$-dependency from the spatial variations of $T(x)$ and $\rho(x)$. At equilibrium, $\mu$ is uniform across the system, and Fig. 1C shows its values as a function of $T$ in that case.

Each simulation was run on 16 processors $(2.5-\mathrm{GHz}$ 4×quad-core AMD Opteron 8380) simultaneously on the Brutus cluster of the ETH Zurich (http://www.cluster.ethz.ch), and took approximately 3 weeks.

\section{ACKNOWLEDGMENTS}

We thank Profs. Signe Kjelstrup and Dick Bedeaux for insightful discussions. Support provided by the European Commission through the MODIFY (FP7-NMP-2008-SMALL-2, Code 228320) research project is greatly acknowledged.
[1] D. A. Edwards, H. Brenner, and D. T. Wasan, Interfacial Transport Processes and Rheology, Butterworth-Heinemann Series in Chemical Engineering (Butterworth-Heinemann, Boston, 1991).

[2] R. Gatignol and R. Prud'homme, Mechanical and Thermodynamical Modeling of Fluid Interfaces, Series on Advances in Mathematics for Applied Sciences (World Scientific, Singapore, 2001).

[3] J. C. Slattery, L. M. C. Sagis, and E.-S. Oh, Interfacial Transport Phenomena, 2nd ed. (Springer, New York, 2007).

[4] S. Kjelstrup and D. Bedeaux, Non-Equilibrium Thermodynamics of Heterogeneous Systems, Series on Advances in Statistical Mechanics (World Scientific, Singapore, 2008).

[5] L. M. C. Sagis, Rev. Mod. Phys. 83, 1367 (2011).

[6] J. S. Rowlinson and B. Widom, Molecular Theory of Capillarity (Dover, Mineola, 2002).

[7] J. D. van der Waals, J. Stat. Phys. 20, 200 (1979).
[8] H. B. Callen, Thermodynamics and an Introduction to Thermostatistics, 2nd ed. (Wiley, New York, 1985).

[9] D. J. Korteweg, Arch. Néerl. Sci. Exactes Nat. 6, 1 (1901).

[10] J. W. Cahn and J. E. Hilliard, J. Chem. Phys. 28, 258 (1958).

[11] H. T. Davis and L. E. Scriven, in Advances in Chemical Physics, edited by I. Prigogine and S. A. Rice (Wiley, New York, 1982) pp. 357-454.

[12] D. M. Anderson, G. B. McFadden, and A. A. Wheeler, Annu. Rev. Fluid Mech. 30, 139 (1998).

[13] A. G. Lamorgese, D. Molin, and R. Mauri, in Multiphase Microfluidics: The Diffuse Interface Model, edited by R. Mauri (Springer, Vienna, 2012) pp. 1-72.

[14] L. Gránásy, T. Pusztai, T. Börzsönyi, J. A. Warren, and J. F. Douglas, Nat. Mater. 3, 645 (2004).

[15] W. J. Boettinger, J. A. Warren, C. Beckermann, and A. Karma, Annu. Rev. Mater. Res. 32, 163 (2002).

[16] V. V. Khatavkar, P. D. Anderson, and H. E. H. Meijer, J. Fluid 
Mech. 572, 367 (2007).

[17] A. Carlson, M. Do-Quang, and G. Amberg, Phys. Fluids 21, 121701 (2009).

[18] J. W. Gibbs, The Scientific Papers of J. W. Gibbs - Vol. I: Thermodynamics (Longmans, London, UK, 1906).

[19] R. C. Tolman, J. Chem. Phys. 16, 758 (1948).

[20] J. G. Kirkwood and F. P. Buff, J. Chem. Phys. 17, 338 (1949).

[21] F. P. Buff and F. H. Stillinger Jr, J. Chem. Phys. 25, 312 (1956).

[22] E. M. Blokhuis and D. Bedeaux, J. Chem. Phys. 95, 6986 (1991).

[23] P. A. Kralchevsky, J. C. Eriksson, and S. Ljunggren, Adv. Colloid Interface Sci. 48, 19 (1994).

[24] M. A. Lomholt and L. Miao, J. Phys. A: Math. Gen. 39, 10323 (2006).

[25] J. R. Lu, R. K. Thomas, and J. Penfold, Adv. Colloid Interface Sci. 84, 143 (2000).

[26] C. V. Sternling and L. E. Scriven, AIChE J. 5, 514 (1959).

[27] D. Bedeaux, E. Johannessen, and A. Røsjorde, Physica A 330, 329 (2003).

[28] E. Johannessen and D. Bedeaux, Physica A 330, 354 (2003).

[29] K. S. Glavatskiy and D. Bedeaux, Phys. Rev. E 77, 061101 (2008).

[30] K. S. Glavatskiy and D. Bedeaux, Phys. Rev. E 79, 031608 (2009).

[31] A. Røsjorde, D. W. Fossmo, D. Bedeaux, S. Kjelstrup, and B. Hafskjold, J. Colloid Interface Sci. 232, 178 (2000).

[32] A. Røsjorde, S. Kjelstrup, D. Bedeaux, and B. Hafskjold, J. Colloid Interface Sci. 240, 355 (2001).

[33] M.-L. Olivier, J.-D. Rollier, and S. Kjelstrup, Colloids Surf. A 210, 199 (2002).

[34] J.-M. Simon, S. Kjelstrup, D. Bedeaux, and B. Hafskjold, J. Phys. Chem. B 108, 7186 (2004).

[35] J. Ge, S. Kjelstrup, D. Bedeaux, J.-M. Simon, and B. Rousseau,
Phys. Rev. E 75, 061604 (2007).

[36] I. Inzoli, S. Kjelstrup, D. Bedeaux, and J.-M. Simon, Chem. Eng. Sci. 65, 4105 (2010).

[37] T. Savin, K. S. Glavatskiy, S. Kjelstrup, H. C. Öttinger, and D. Bedeaux, Europhys. Lett. 97, 40002 (2012).

[38] H. C. Öttinger and D. C. Venerus, AIChE J. 60, 1424 (2014).

[39] S. Kjelstrup, D. Bedeaux, I. Inzoli, and J.-M. Simon, Energy 33, 1185 (2008).

[40] B. Widom, J. Chem. Phys. 39, 2808 (1963).

[41] D. Frenkel and B. Smit, Understanding Molecular Simulation: from Algorithms to Applications, 2nd ed. (Academic Press, San Diego, 2002).

[42] It is worth observing that latent heat produced by the perpetual conversion of vapor into liquid appears to flow away from the interface into the bulk phases, following the opposite gradients of temperature featured in the liquid and vapor phases constrained to mass flow (see Fig. 3B). Dissipative processes of mass and heat transfer at phase boundaries are highly coupled, and we notably observe a mass transfer from the cold to the warm regions in the vapor phase [48].

[43] V. K. Badam, V. Kumar, F. Durst, and K. D. Danov, Exp. Therm. Fluid Sci. 32, 276 (2007).

[44] F. Duan, C. A. Ward, V. K. Badam, and F. Durst, Phys. Rev. E 78, 041130 (2008).

[45] F. E. G. Güner, J. Wåhlin, M. Hinge, and S. Kjelstrup, Chem. Phys. Lett. 622, 15 (2015).

[46] J. Xu, S. Kjelstrup, D. Bedeaux, A. Røsjorde, and L. Rekvig, J. Colloid Interface Sci. 299, 452 (2006).

[47] D. Jou, J. Casas-Vázquez, and G. Lebon, Extended Irreversible Thermodynamics, 4th ed. (Springer, Dordrecht, 2010).

[48] H. Struchtrup, S. Kjelstrup, and D. Bedeaux, Phys. Rev. E 85, 061201 (2012). 